\title{
Some properties of auditory memory for rapid formant transitions
}

\author{
PETER HOWELL \\ University College London, Gower Street, London, England WCIE 6BT \\ and \\ CHRISTOPHER J. DARWIN \\ University of Sussex, Falmer, Brighton, England BNI $9 Q G$
}

\begin{abstract}
In the three experiments reported here, subjects indicate whether two sequentially presented syllables, differing in the place of articulation of an initial stop consonant, are phonemically the same or not. The first experiment finds faster "same" responses to acoustically identical pairs than to pairs that are phonemically identical but acoustically distinct, provided that the second syllable is presented within $400 \mathrm{msec}$ of the first. This is interpreted as indicating the persistence of a memory which preserves auditory information about within-category distinctions. The third experiment shows that this advantage remains when a tone is interposed between the two syllables, but is removed when a brief vowel is similarly interposed. The second experiment presents the second syllable of each pair dichotically with a noise burst, and shows that the size of the right-ear advantage for this reaction time task is reduced when the result of comparisons based on this auditory memory is compatible with the required phonemic decision, but that the right-ear advantage is increased when auditory comparisons would contradict the phonemic relationship.
\end{abstract}

Although the concept of a memory containing uncategorized auditory information has been invoked to explain a variety of phenomena in the perception of speech and of other sounds (Darwin \& Baddeley, 1974; Fujisaki \& Kawashima, 1968; Massaro, 1970; Pisoni, 1973), evidence for its existence has generally been confined to sets of sounds which could be distinguished by gross auditory attributes (Darwin \& Baddeley, 1974; Darwin, Turvey, \& Crowder, 1972; Massaro, 1970), leaving open the possibility that the fine auditory detail responsible, for example, for distinguishing the place of articulation of a stop consonant is precluded from such a memory (Liberman, Mattingly, \& Turvey, 1972; Repp, Note 1). This paper aims to demonstrate that this fine auditory information is preserved and also that it decays with time.

The main evidence for decay in auditory memory comes from the delayed partial-sampling technique (Darwin, et al., 1972; Treisman \& Rostron, 1972), since neither the effect of a delayed suffix on recency (Crowder, 1973) nor the decreased backward masking of one sound by another with increased temporal

We would like to thank the Science Research Council for supporting the first author on a studentship and the second with a research grant. We would like also to thank Professor R. J. Audley, Dr. A. J. Fourcin, and A. C. Davis for useful comments. Computing facilities for stimulus preparation at Sussex were provided by the Medical Research Council. Part of this work was reported at the 19th Tagung Experimentell Arbeitenden Psychologen at Konstanz, March 1977. separation (Massaro, 1970) can distinguish between decay of a memory and the time course of readout from it. Unfortunately, the auditory partial-sampling technique itself is limited in its value here, since it is not clear from published experiments whether or not the memory whose decay it is demonstrating is one prior to phonemic categorization.

The technique that gives the clearest evidence for a memory preserving auditory information prior to phonemic categorization is the extension of Posner's letter-matching paradigm due to Pisoni and Tash (1974). Their procedure compares the time taken to classify consecutively presented pairs of syllables as being phonemically the same or different according to whether the pair is acoustically identical or not, and according to the degree of acoustic difference between them. This procedure is the analog of a visual letter-matching experiment in which subjects might be asked to indicate whether consecutively presented letters have the same name and their reaction time is measured as a function of the physical similarity of letter pairs having either the same or different names. Pisoni and Tash found that pairs of syllables which differed slightly in voice-onset time along a $/ \mathrm{ba} /-/ \mathrm{pa} /$ continuum, but which fell within the same phoneme category and so were virtually indistinguishable on a discrimination test, were judged "same" slower than pairs that were acoustically identical. They also found that "different" judgments were faster the greater the acoustic difference between the syllables being compared. Both these effects 
they ascribed to an auditory memory persisting from the presentation of the first syllable to that of the second. The model they proposed to account for their results is similar to that used, for example, by Atkinson and Juola (1973) to account for word recognition in a memory-scanning paradigm. Essentially, the model allows a subject to judge two syllables to be the same if they are sufficiently similar or different if they are sufficiently distinct according to some metric of acoustic similarity. If their similarity falls between these two criteria, then slower, more analytic mechanisms must be used to decide whether they are phonemically different. Acoustically identical and acoustically very distinct syllables can thus be judged the same or different, respectively, by rapid processes using auditory dimensions of similarity. Phonemically identical syllables which are not acoustically identical and phonemically different syllables which are acoustically similar, on the other hand, must be categorized phonemically before the subject can make a reliable decision about their phonemic relationship. According to this model, any effect of acoustic distance on either "same" or "different" responses reflects the presence of an auditory memory, which from the Pisoni and Tash (1974) experiment must persist for at least $550 \mathrm{msec}$, since syllables of $300-\mathrm{msec}$ duration were separated by $250 \mathrm{msec}$ of silence, giving a stimulus onset asynchrony (SOA) of $550 \mathrm{msec}$.

Eimas and Miller (Note 2) have extended this work to a place-of-articulation continuum $(/ \mathrm{ba} /-/ \mathrm{da} /)$, looking for evidence of decay in auditory memory by varying the time interval between the two syllables to be compared. They found, using $250-\mathrm{msec}$ syllables, that for "same" responses acoustically identical syllables gave faster reaction times at SOAs of 300 and $500 \mathrm{msec}$ but not at $1,050 \mathrm{msec}$. However, they also found that the faster "different" reaction time to acoustically more different syllables persisted even at long SOAs.

Repp (1975), using a voice-onset time continuum and presenting each member of a pair to a different ear, has also found faster "different" reaction times to acoustically more different pairs, which again did not change significantly with changing delay. Both he (Repp, Note 1) and Eimas and Miller (Note 2) pointed out that, since acoustically more similar "different" pairs tend to have one or both members closer to the phoneme boundary than do acoustically less similar pairs, the slower reaction time to the former could be due to the slower categorization time found for sounds close to a phoneme boundary (Pisoni \& Tash, 1974; Studdert-Kennedy, Liberman, \& Stevens, 1963; Eimas \& Miller, Note 2). In addition, both in this study and a later one (Repp, Note 1), which used both binaural and alternating monaural presentation as well as a modified design to circumvent the confuunding of acoustic distance with proximity to the phoneme boundary, Repp failed to find a significantly faster reaction time for acoustically identical same responses than for nonidentical same responses. This failure $\mathrm{n}$ ' 'ly be due to his collecting insufficient data, since there were nonsignificant tendencies in the appropriate direction.

Two main issues emerge from these results. First, are the claims of quicker reaction times for same judgments made to acoustically identical sounds than for those made to nonidentical sounds reliable, and does this effect disappear with increasing SOA? Second, does the effect of acoustic disparity on different judgments also reflect an auditory memory, as Pisoni and Tash (1974) maintained, or is it merely an artifact of the proximity to a phoneme boundary of the second syllable of a pair? If the latter is the case, then we would expect to find no attenuation of this effect with increasing SOA.

An alternative notion of how same-different judgments for syllables are made, proposed by Morais and Darwin (1974), makes rather different predictions from the Pisoni and Tash (1974) model. Morais and Darwin found smaller ear differences and quicker overall responses for same than for different judgments on a syllable-matching task (see also Darwin, Howell, \& Brady, in press; Morais, 1975). This led them to suggest that the same judgments could be made on the basis of an auditory comparator which was less lateralized than the subsequent, and therefore slower, processes of categorization and comparison needed for different judgments. Their suggestion was based on a two-stage model proposed by Bamber (1969) for the comparison of letter strings. A number of subsequent experiments have supported Bamber's notion of two stages, by showing that same and different judgments are differentially affected by different factors (e.g., Besner \& Coltheart, 1975; Krueger, 1973), although it is likely that factors other than physical identity contribute in some cases to same responses being faster than different responses (Bamber, 1972; Bamber \& Paine, 1973; Howell \& Stockdale, 1975).

The Morais and Darwin (1974) model, unlike that of Pisoni and Tash (1974), predicts that auditory memory should not be used for different judgments, but, like that of Pisoni and Tash, claims that the categorization process is needed for same judgments which are not acoustically identical. Both models imply that same judgments to acoustically nonidentical sounds should be as slow as different judgments, since neither can use the fast auditory comparator, and also that for pairs judged the same, any effect of proximity to a phoneme boundary of the second stimulus should appear only for acoustically nonidentical pairs, since the acoustically identical ones do not need the phonemic categorization process.

Our first experiment is based on the Eimas and Miller (Note 2) study, but uses a /ba/-/ga/ difference rather than a $/ \mathrm{ba} / / \mathrm{da} /$. This increases the acoustic 
Table 1

Number of Occurrences of the Stimulus Pairs Used in Experiment 1

\begin{tabular}{|c|c|c|c|c|c|}
\hline & \multicolumn{2}{|c|}{ "Same" Pairs } & \multicolumn{3}{|c|}{ "Different" Pairs } \\
\hline & Identical & Nonidentical & 8 Step & 10 Step & 12 Step \\
\hline & $\begin{array}{c}1-1 \\
3-3 \\
11-11 \\
13-13\end{array}$ & $\begin{array}{c}1-3 \\
3-1 \\
11-13 \\
13-11\end{array}$ & $\begin{array}{l}3-11 \\
11-3\end{array}$ & $\begin{array}{c}1-11 \\
11-1 \\
3-13 \\
13-3\end{array}$ & $\begin{array}{c}1-13 \\
13-1\end{array}$ \\
\hline $\begin{array}{l}\text { Equal Pair } \\
\text { Equal Step }\end{array}$ & $\begin{array}{l}9 \\
9\end{array}$ & $\begin{array}{l}9 \\
9\end{array}$ & $\begin{array}{r}9 \\
12\end{array}$ & $\begin{array}{l}9 \\
6\end{array}$ & $\begin{array}{r}9 \\
12\end{array}$ \\
\hline
\end{tabular}

Note-The stimulus pairs were presented equally often at each of the stimulus onset asynchronies. The numbers in the last two rows are the number of trials in each block for each of the above stimulus pairs in the two probability conditions of Experiment 1.

distance between pairs in terms of the second formant transition trajectory (which carries the main load of place-of-articulation information in front of $/ a /$ ). Subjects might therefore be more willing to use auditory information for different judgments if the Pisoni and Tash (1974) model is correct in allowing them to do this. We have also designed the experiment to allow the effects of acoustic distance between syllable pairs and proximity of the second syllable of a pair to the phoneme boundary to be analyzed separately.

\section{EXPERIMENT 1}

\section{Method}

Subjects. Eight undergraduate subjects (six female and two male) attended for three sessions of approximately $1.5 \mathrm{~h}$ each. They were between 19 and 23 years of age, were all right handed, and reported no history of hearing defects. They were paid $\& 1$ per session.

Stimuli. Each of the 13 stimuli on the /ba//da//ga/ continuum used here lasted $200 \mathrm{msec}$, with the initial consonant being cued by linear $40-\mathrm{msec}$ transitions. The first formant always started at $150 \mathrm{~Hz}$ and rose linearly (in Hertz) to its steady-state value of $743 \mathrm{~Hz}$. The steady-state values for Formants 2 and 3 were $1,620 \mathrm{~Hz}$ and $2,862 \mathrm{~Hz}$. These were again approached linearly by transitions whose starting points were arranged in equal steps between the following positions. The starting point for Stimulus 1 was $1,232 \mathrm{~Hz}$ for the second formant and $2,181 \mathrm{~Hz}$ for the third. The second-formant starting points increased in $77-\mathrm{Hz}$ steps up to $2,156 \mathrm{~Hz}$ for Stimulus 13, and those for the third formant increased in 169-Hz steps up to $3,159 \mathrm{~Hz}$ for Stimulus 7 and then decreased again in $169-\mathrm{Hz}$ steps back down to $2,181 \mathrm{~Hz}$ for Stimulus 13 . These 13 sounds, together with a $1 \mathrm{kHz}$ warning tone, were computed on a software parallel formant synthesizer running on a PDP-12 computer, stored on a disk, and then output through 12-bit digital-to-analog converters at $8 \mathrm{kHz}$, low-pass filtered at $4 \mathrm{kHz}$, and then recorded in experimental sequences on a Revox tape recorder. All the stimuli in this experiment were presented binaurally to subjects at approximately $75 \mathrm{~dB}$ SPL, and all the syllables had a constant pitch of $120 \mathrm{~Hz}$.

Identification test. Two identification sequences, both randomizations of 10 tokens of each of the 13 stimuli, were prepared. A trial consisted of the $1 \mathrm{kHz} 24-\mathrm{msec}$ warning tone, followed after a 500-msec pause by a stimulus to be identified, and then a 3 -sec pause.

Before each session, subjects received one 130-trial identification test. Subjects classified each syllable as $/ \mathrm{ba} /, / \mathrm{da} /$, or $/ \mathrm{ga} /$ by pressing one of three response keys using three fingers of one hand.
Stimulus-matching task. In the stimulus-matching task, the subject indicated whether or not the second syllable had the same initial consonant as the first. He did this by pressing one of two response keys, one below each index finger. The response made and its reaction time (RT) were recorded by a PDP-1 2 computer.

Stimuli $1,3,11$, and 13 were used in the same-different matching task. These were chosen on the basis of pilot experiments as being readily identifiable as either / ba/ ( 1 and 3) or /ga/ (11 and 13). Each stimulus was paired with every other an equal number of times in the equal pair probability condition. This led to an unequal number of 8-, 10-, and 12-step different pairs, so for the equal step probability condition the number of 10-step pairs was reduced to be equal to the 8- and 12-step pairs. The number of trials per block for each of the various pairs in the conditions are shown in Table 1.

A trial consisted of the warning tone followed after a 500 -msec pause by the first syllable. This was then followed by a $50-; 200-$, or 800 -msec pause, after which came the second syllable. This gave SOAs of 250,400 , and $1,000 \mathrm{msec}$, which were randomized within a block of 144 trials. A pause of $3 \mathrm{sec}$ separated trials. Two randomizations of 144 trials in each probability condition were prepared.

Each subject did one practice and two experimental sessions, each of which consisted of all four of these 144-trial blocks. The two blocks from a particular probability condition were always given consecutively, but which probability condition came first was counterbalanced between the two experimental sessions and between subjects. Which hand was used to indicate "same" was counterbalanced between subjects.

\section{Results}

The response categories to which subjects assigned the 13 sounds of the identification test are shown in Figure 1. None of the stimuli used in the matching task

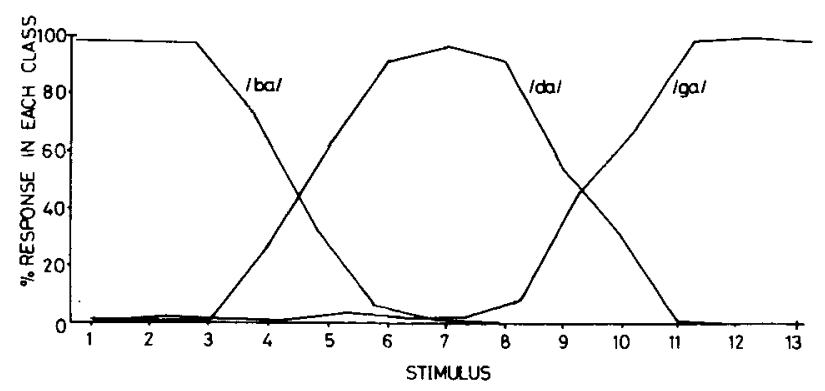

Figure 1. Percent identifications of the 13 stimuli used as /ba/, /da/, or /ga/ by subjects in Experiment 1. Stimuli 1, 3, 11, and 13 were used in the matching experiment. 

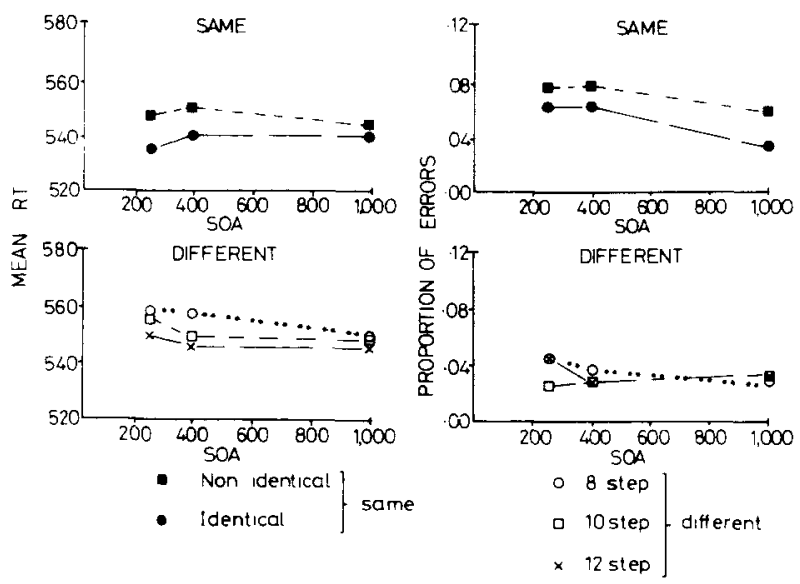

Figure 2. Mean correct reaction times and percent errors for same-different judgments (on the basis of phonemic identity) of syllable pairs in Experiment 1, as a function of the stimulus onset asynchrony of the pair of syllables to be compared. Same pairs are either acoustically identical or nonidentical, and different pairs can differ in the number of stimulus steps separating them acoustically.

were assigned more than $2 \%$ of the time to a category other than that required for phoneme matching to give the expected response.

For the same-different matching task, mean correct RTs and proportion of errors for each condition were calculated for each block of 144 trials. Reaction times of less than $100 \mathrm{msec}$ or longer than $1 \mathrm{sec}$ were not included in the analysis, and are not included in the mean RTs and errors across the eight subjects shown in Figure 2. These accounted for $3.6 \%$ of the trials.

In all the analyses reported in this paper, the means of the RTs by blocks are used untransformed, but the error data are averaged by block and then arc-sine root transformed. Separate six-way analyses of variance were carried out on the RTs and errors for same and different responses. These distinguished as factors: subjects, probability conditions, sessions, blocks, SOA, and matching conditions (i.e., identical vs. nonidentical for the same judgments and step size for the different judgments).

For the RTs of same responses, there was an interaction between matching condition and SOA $[F(2,14)=4.5, p<.05]$. This interaction arose, as individual $t$ tests showed, because same responses to acoustically identical stimuli were faster than those to nonidentical at the $250-\mathrm{msec}$ SOA $[\mathrm{t}(7)=5.4, \mathrm{p}<.001]$ and the $400-\mathrm{msec}$ SOA $[\mathrm{t}(7)=4.8, \mathrm{p}<.01]$, but not at the $1,000-\mathrm{msec}$ SOA $[\mathrm{t}(7)=1.7, \mathrm{p}>.10]$. There was a main effect of matching condition $[F(1,7)=16.9$, $p<.005]$. There was also an interaction between sessions and blocks $[F(1,7)=6.6, p<.01]$, through subjects speeding up less during the second than the first day. Analysis of the "different" RTs showed an interaction between matching conditions and SOA $[F(4,28)=3.2, p<.05]$ and a large main effect of matching condition $[\mathrm{F}(2,14)=28.5, \mathrm{p}<.001]$.
For errors to same stimulus pairs, there was a main effect of SOA $[F(2,14)=4.9, p<.05]$ and of matching condition $[F(1,7)=8.0, p<.05]$, but no interaction between them $(F<1)$. These effects were due to there being more errors in the nonidentical matching condition and at short SOAs. Analysis of errors to different stimulus pairs showed no significant effect nor any significant interaction.

Since the interaction between SOA and matching condition for the different response RTs was very weak compared with the size of the main effect of matching condition (and with hindsight from the next experiment), we examined whether the main effect of matching condition found in this experiment was attributable to the step size separating the pairs, or rather to the proximity of the second syllable to a phoneme boundary. We therefore looked separately at pairs differing by a constant 10 steps to see if there was any difference between them depending on the distance of the second syllable from the phoneme boundary. This analysis showed a significant effect of boundary distance on RTs $[F(1,21)=12.8, p<.005]$, but none on errors. To see whether this boundary effect could explain the apparent effect of step size, we then analyzed for step size while keeping distance of the second syllable from the boundary constant. This analysis showed that there was no significant residual effect of step size on the different judgments $[F(3,21)=2.4, p>.05]$. Note that the effect of matching condition on same judgments cannot be explained away by this artifact, and indeed there was no effect of proximity to the phoneme boundary for identical pairs $[F(1,7)=1.2, p>.10]$. However, neither was there any effect of proximity to the phoneme boundary for the nonidentical pairs $[F(1,7)=2.7$, $\mathrm{p}>.10]$. A further analysis showed no significant difference in reaction time between the nonidentical same judgments and the average different judgments $[F(1,7)=2.7, \mathrm{p}>.10]$.

\section{Discussion}

This experiment has extended to place of articulation the Pisoni and Tash (1974) finding that pairs of acoustically identical syllables are judged phonemically the same faster than those that are not acoustically identical; this confirms an earlier report by Eimas and Miller (Note 2). Like Eimas and Miller, we also have found that this advantage for the acoustically identical stimulus pairs disappears with increasing SOA. Our experiment confirms that the formant transition information subserving place of articulation for stops is preserved in an auditory memory for at least $400 \mathrm{msec}$ but for less than $1 \mathrm{sec}$.

We have also shown that the longer RT to phonemically different pairs that are acoustically similar is due as both Eimas and Miller (Note 2) and Repp (1975, Note 1) have suggested, to the second stimulus of a pair being more likely to be close to a phoneme 
boundary, rather than, as Pisoni and Tash originally suggested, to the acoustic distance separating the syllable pair. Although this result is strong evidence against their model, the interaction of matching condition with SOA found for both the same and the different pairs is somewhat equivocal. We have chosen to stress the interaction for same pairs at the expense of the almost equally strong one for different pairs, since, although the latter interaction is not found in the next experiment, the interaction for same judgments is strongly replicated. Further discussion of the relative success of the two models is postponed until after the next experiment.

The next experiment is basically a repeat of Experiment 1 , but, by presenting the second stimulus of a pair dichotically. together with a contralateral noise burst, examines the interaction of auditory memory effects with ear differences. According to the Morais and Darwin (1974) model, ear differences for same judgments to acoustically identical sounds should be smaller than those to acoustically nonidentical sounds and for different judgments at short SOAs, but the ear differences should converge at longer SOAs.

\section{EXPERIMENT 2}

\section{Method}

Subjects. Eight right-handed subjects paid at the same rate as in I:xperiment 1 attended for five sessions of about $1.5 \mathrm{~h}$ each. Their ages were between 19 and 28 and none reported any hearing defects.

Procedure. The stimulus sequences used here were similar to those used in Experiment 1 , with the warning tone and first syllable presented binaurally; but the second syllable on each trial of the syllable-matching experiment was presented to one ear simultaneously with a $240-\mathrm{msec} 60-\mathrm{dB}$ SPL burst of $4-\mathrm{kHz}$ low-pass filtered noise to the opposite ear. The second syllable was played to the same ear throughout a block of 144 trials.

In the matching task, the subject used a lever which he moved toward or away from him to signal "same" or "different." The direction of movement for, say, "same" was fixed for a subject but was counterbalanced between subjects., As in Experiment 1, each session had four blocks of 144 trials, but the number of experimental sessions was doubled to allow headphone orientation, the channel the second syllable was played to, and the hand used to operate the lever to be counterbalanced across blocks and subjects. The first session began with the same identification task used in Experiment 1.

\section{Results}

The results of the identification task were similar to those shown in Figure 1 with somewhat sharper category boundaries and indicated that no stimulus used in the matching task was assigned more than $5 \%$ of the time to other than the expected response category. The data were treated in the same manner as in Experiment 1, and the mean RTs and error rates are shown in Figures 3 and 4 . Reaction times outside the analyzed range of 100 to $1,000 \mathrm{msec}$ accounted for $4.3 \%$ of the data.

Statistical analysis was carried out in a similar way to

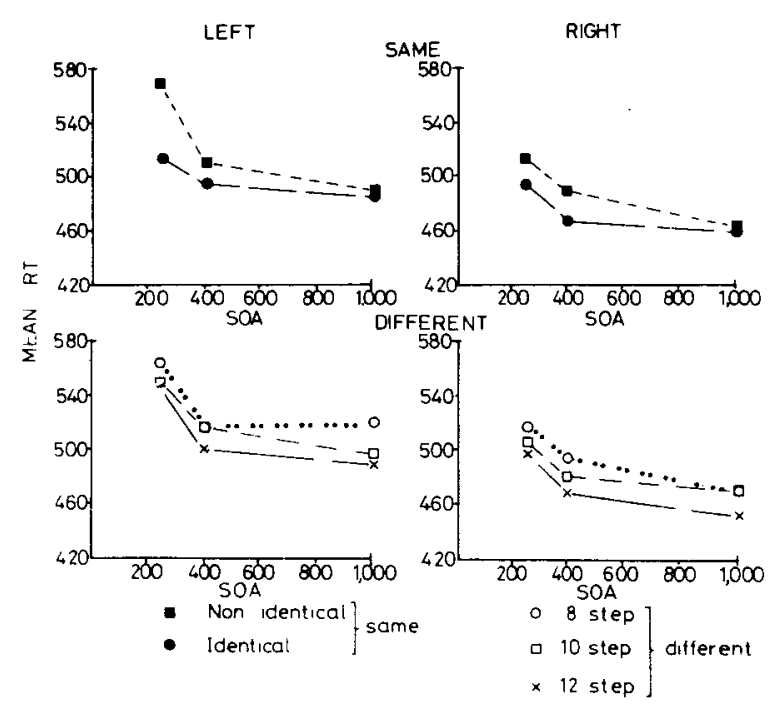

Figure 3. Mean correct reaction times for the syllablematching task in Experiment 2, as a function of stimulus onset asynchrony and the ear receiving the second syllable. Presentation of the latter syllable was dichotic with a contralateral noise burst.

the last experiment, but with the additional factor of cars being added to the analyses. For RTs of same responses, the main effects of ear $[F(1,7)=5.7$, $\mathrm{p}<.05]$, SOA $[\mathrm{F}(2,14)=10.5, \mathrm{p}<.01]$, and matching condition $[F(1,7)=24.7, p<.01]$ were significant, as were the interactions of SOA by Matching Condition $[F(2,14)=7.7, p<.01]$, Ear by SOA by Matching Condition $[F(2,14)=4.0, p<.05]$, and Sessions by Ear by SOA by Matching Condition $[F(2,14)=6.3, p<.05]$. Thus, stimuli going to the right ear were reacted to quicker than those going to the left, and RTs were longer at shorter SOAs. Responses to identical stimuli were quicker than those

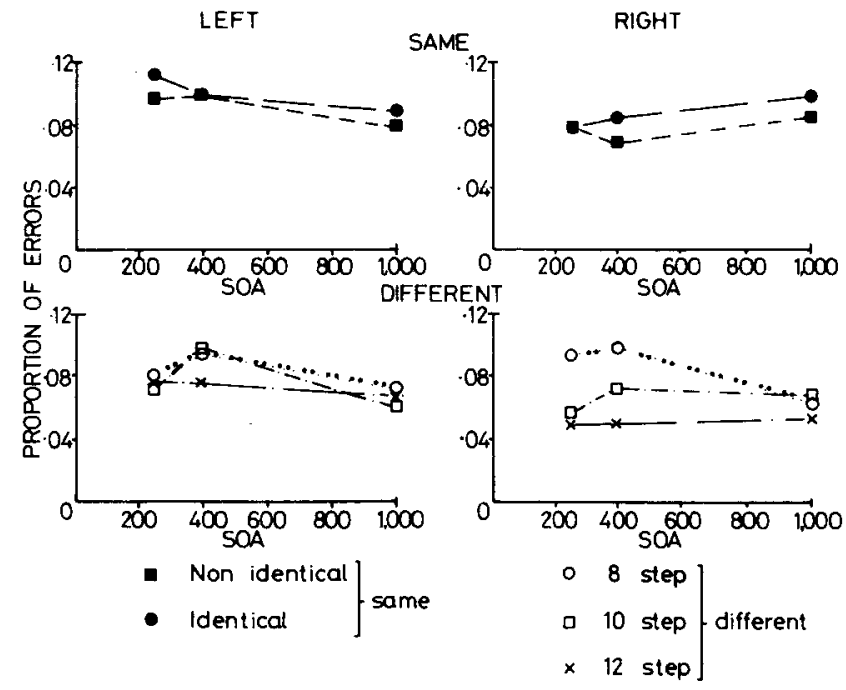

Figure 4. Mean percent errors for the syllable-matching task in Experiment 2. 
to nonidentical stimuli, and this difference was more pronounced at shorter SOAs for stimuli arriving at the left ear. Finally, the last effect attenuated over sessions.

The interaction of the acoustic identity advantage with ear and SOA was examined in more detail by doing individual $t$ tests at each SOA for each ear. These showed that the acoustically identical pairs were responded to faster than the nonidentical pairs on each ear at the two shorter SOAs, but that there wis no difference at the longest SOA. Moreover, the size of the acoustic advantage was larger on the left ear than on the right at the $200-\mathrm{msec}$ SOA, but not at the two longer intervals (all tests significant at $p<.001$ ).

On the RTs for the different responses, ear $[F(1,7)=17.5, \quad p<.01], \quad$ SOA $\quad[F(2,14)=43.4$, $\mathrm{p}<.001]$, and matching condition $[\mathrm{F}(2,14)=14.3$, $\mathrm{p}<.001]$ reached significance, as well as the Session by Block interaction $[F(3,21)=3.1, p<.05]$. Syllables going to the right ear were responded to quicker than those to the left, larger acoustic differences between different pairs gave quicker RTs, and the decrease in RT within a session was smaller in later sessions.

The corresponding analyses on errors showed that for those made on "same" trials, more were in earlier sessions $[F(3,21)=3.8, p<.05]$, and an increase in errors for sounds on the left ear was more marked at shorter SOAs $[F(2,14)=8.7, p<.01]$. Analysis of the errors to "different" pairs showed a main effect of SOA $[\mathrm{F}(2,14)=6.6, \quad \mathrm{p}<.01]$ and matching condition $[F(2,14)=9.9, p<.01]$, and significant interactions between ear and sequence $[F(3,21)=3.3, p<.05]$. The "different" RTs were analyzed, as in Experiment 1, to separate the effects of acoustic step size and those of proximity to the phoneme boundary of the second syllable. Again, there was a highly significant effect of proximity to the boundary $[F(1,21)=9.8$, $\mathrm{p}<.001]$, but also some slight residual effect of step size $[F(3,21)=3.4, p<.05]$.

\section{Discussion}

The main results of the first experiment have been replicated here. In particular, the advantage for acoustically identical pairs and its attenuation with time has been demonstrated separately for signals to the left and right ear; by contrast, the effect of step size (or rather of proximity to the phoneme boundary) on the "different" responses does not, in this experiment, attenuate with increasing SOA. This justifies our earlier dismissal of the weak interaction found in Experiment 1.

We will now examine in detail how well the two models described in the introduction handle the combined results of the two experiments. Their main distinguishing feature is that the Pisoni and Tash (1974) model allows comparisons based on the contents of auditory memory to influence both same and different responses, whereas the Morais and Darwin (1974) model confines different judgments to information at a categorical level, and same judgments of acoustically identical stimuli to information at the auditory level.

When the large effect found in both experiments (both ps $<.005$ ) of proximity of the second syllable to a phoneme boundary is controlled for, there is only weak evidence for any residual effect of acoustic distance on different judgments (Experiment 1, p > .05; Experiment 2, .05>p> .025). Absence of this effect is predicted by the Morais and Darwin (1974) model. The absence from both experiments of a systematic convergence of RTs to different pairs that differ to a greater or smaller acoustic extent as SOA increases argues also against the same form of information being used for the different judgments as for the same judgments.

A further test of differences between the two models comes from a comparison between nonidentical same and different responses. As predicted by the Morais and Darwin (1974) model, neither experiment showed any difference between the two RTs (both ps $>.10$ ).

Two further predictions of both models should be considered, although they do not distinguish them. If identical same judgments are being made on the basis of auditory information, then they should be no slower when the sounds to be compared fall close to a phoneme boundary than when they do not. This is true for both experiments $(p>.10)$. But, similarly, if nonidentical same judgments cannot be made on the basis of acoustic identity, then they should be slower when the second syllable comes close to a phoneme boundary. This turns out not to be the case in either experiment $(p>.10)$.

The pattern of ear differences between the three main conditions is generally explicable in terms of the Morais and Darwin (1974) model. At the shortest SOA, there is a smaller ear difference for the acoustically identical matches than for either the acoustically nonidentical same judgments or the different judgments. This, we would argue, is because the same judgments for acoustically identical pairs can be made on the basis of an auditory comparator, which is less lateralized than the process of postcategorical comparison. At longer SOAs, though, the auditory component is no longer usable and so recourse must be made to categorical comparisons, leading to similar ear differences for both types of same judgments and different judgments.

An interesting anomaly is the unusually large ear difference for the same judgments to acoustically nonidentical sounds at the shortest SOA. In this condition, there is most likely to be conflict between the output of an auditory comparator and a phonemic comparator, since although the correct response is "same," there is still sufficient auditory information available about the first syllable for its nonidentity to the second to be apparent (as borne out by the quicker overall reaction time for the identical match). Such conflict might put more of a burden on the categorical 
component, and so give a greater advantage to sounds having readier access to this presumably left-hemisphere process. This situation is analogous to the Stroop paradigm, which is known to give larger ear differences when the irrelevant dimension is in conflict with the response than when it is compatible (Cohen \& Martin, 1975). This aspect of the data is not predicted by the Morais and Darwin (1974) model, since it indicates that the result of an auditory level comparison influences nonidentical judgments.

Having confirmed both the existence of a decaying prephonemic memory for the auditory information subserving place of articulation in stop consonants and also its relationship to laterality effects, we now ask whether, like auditory memories revealed by other paradigms, this store is susceptible to selective interference by subsequent auditory events.

\section{EXPERIMENT 3}

An important property of the auditory memory called precategorical acoustic storage (PAS) by Crowder and Morton (1969) is that it is interfered with by sounds attributable to the same source. By interposing different sounds between the two syllables to be compared in this reaction time paradigm, we can ask what types of sound abolish the advantage for acoustically identical over nonidentical "same" judgments, and by implica. tion, thus interfere with the auditory trace of the first syllable or its availability. The next experiment uses two such "masks," a vowel identical to that used in the syllables and a $1-\mathrm{kHz}$ tone. If this store is the same as PAS, we would expect that the advantage for the identical pairs would be abolished by the vowel mask but not by the tone mask.

\section{Method}

Subjects. Eight undergraduate subjects attended for three sessions, each of about $1 \mathrm{~h}$. They were right handed and between 18 and 24 years of age. None reported any hearing defects, and they had all previously done an experiment involving reaction times to synthetic speech. They were paid 60 pence a session for their participation.

Procedure. The syllables used in this experiment were identical to those used in Experiment 1, except that the steady. state vowel was reduced to make the whole syllable $100 \mathrm{msec}$ long. The identification test used in this experiment was identical to that used previously. In the matching experiment, two additional sounds were used, a $100-\mathrm{msec}$ vowel identical to that used for the steady state of the syllables with the same amplitude contour as the whole syllable, and a $1 \mathrm{kHz} 75-\mathrm{dB}$ SPL tone also lasting $100 \mathrm{msec}$ and similarly contoured.

There were three different types of trials, depending on the event intervening between the two syllables to be compared. These syllables were always separated by a $400-\mathrm{msec}$ SOA, but whereas in the "silence" condition the intervening $300 \mathrm{msec}$ was silent, in the "vowel" and "tone" conditions the appropriate mask was played separated from each syllable by $100 \mathrm{msec}$. The three different types of trials occurred in a random order within blocks, separated by a 2 -sec pause.

Each subject took one practice and two experimental sessions of three blocks of 192 trials. Only the equal pair probability condition was used in this experiment, since neither of the previous ones had shown any effect of probability condition. The method of responding was the same as in Experiment 2, and the counterbalancing procedure was basically the same as in the previous experiments.

\section{Results}

The results of the identification experiment were again similar to those shown in Figure 1, but with rather steeper category boundaries. None of the stimuli used in the matching task was assigned to other than the expected category more than $6 \%$ of the time. Figure 5 gives the mean correct RTs and error rates for the three types of intervening event. Reaction times outside the analyzed range of 100 to $1,000 \mathrm{msec}$ accounted for $2.4 \%$ of the data.

As a check that the advantage for identical same pairs was replicated in the silence condition, a four-way analysis of variance distinguishing subjects, sessions, blocks within a session, and matching conditions was run. This showed a significant effect of matching condition, with identical being faster than nonidentical $[F(1,7)=30.9, p<.001]$, and also an effect of blocks within a session indicating that subjects tended to speed up within a session $[F(2,14)=7.5, p<.01]$. Similar analyses on the different responses and on errors showed no significant effects.

The effect of the intervening event was then examined by five-way analyses distinguishing subjects, sessions, blocks, matching conditions, and silence/vowel/ tone as intervening event. For same responses there was a main effect of intervening event $[\mathrm{F}(2,14)=19.3$, $\mathrm{p}<.001]$, with silence being quicker than vowel and tone, and the predicted interaction between intervening event and matching condition $[\mathrm{F}(2,14)=8.8, \mathrm{p}<.01]$ attributable to a reduced advantage for the identical condition when the vowel intervened. Subsequent four-way analyses on the tone and vowel conditions showed, for same responses, an advantage for the identical match in the tone condition $[F(1,7)=8.1$, $\mathrm{p}<.01]$, but not in the vowel condition $(\mathrm{F}<1)$.

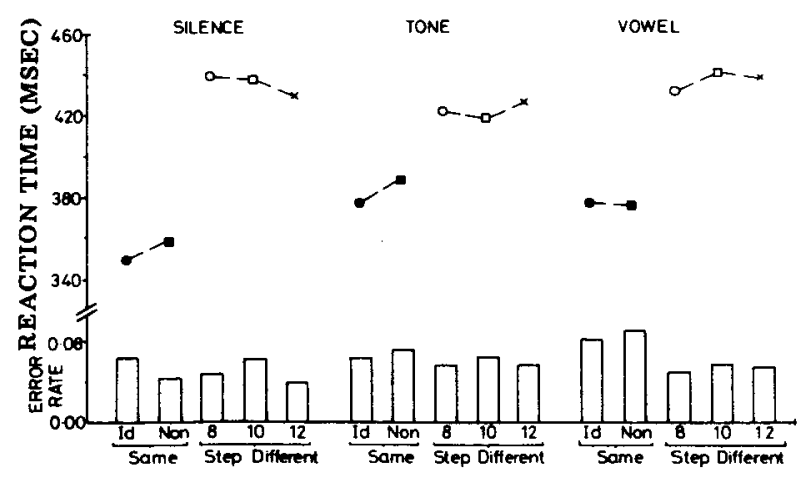

Figure 5. Mean correct reaction times and percent errors for the syllable-matching task in Experiment 3. The three sections refer to the event (silence, $1-\mathrm{kHz}$ tone, or vowel $/ \mathrm{a} /$ ) intervening between the two syllables, whose SOA was always 400 msec. 
For different responses, the main effect of intervening event was significant $[F(2,14)=18.7, p<.001]$, as was the interaction between matching condition and intervening event $[F(2,14)=5.8, p<.01]$. The presence of this interaction is predicted by the Pisoni and Tash (1974) model, but the pattern of the individual data points is not, since, given the selective interference of auditory memory by the vowel sound for same responses, this model predicts a decrease of RT with step size in the tone condition, but no such decrease in the vowel condition.

For error rates to same trials, the main effect of intervening event was significant, indicating more errors when the vowel intervened $[F(1,7)=11.1, p<.05]$, but for error rates to different trials, nothing was significant.

\section{Discussion}

The auditory memory responsible for the advantage shown at short SOAs by acoustically identical over nonidentical syllables can be rendered ineffective by an additional vowel played between the two syllables to be compared. By contrast, it is unaffected by an intervening tone. There is, therefore, an analogy between this memory and PAS, inasmuch as both appear to be susceptible to interference from sounds from the same auditory source. Whether this store is immune when the mask differs along such dimensions as spatial location and voice pitch remains to be seen, as does the question of whether different vowels spoken in the same voice and from the same location cause this interference.

\section{GENERAL DISCUSSION}

In conclusion, we have confirmed the presence of a decaying auditory store which is capable of preserving the fine structure of formant transitions for at least $400 \mathrm{msec}$, and have demonstrated that this information is rendered ineffective by subsequent presentation of a vowel identical to that used in the syllable present in the store, but that it is not influenced by the similar presentation of a tone. We have also provided some evidence that this store is probably not used in determining that two syllables differ phonemically, but that its use is confined to judging that syllables are identical. We have further shown that when this store is used and comparisons from it are compatible with those from the categorical comparison process, smaller ear differences are obtained than when the store is either not used (as in "different" judgments) or when the results of comparisons based on its contents contradict those of the categorical comparison process (as in judging acoustically nonidentical syllables to be the "same").

Neither of the two simple models satisfactorily explains our data. The finding in the last experiment of a differential effect of vowel and tone masks on "different" responses suggests that auditory information may be being used for that judgment; but against this we find no consistent evidence for decrease of the effect of step size on "different" judgments as SOA increases, nor any consistent residual effect of step size, when proximity to a phoneme boundary is controlled for. The pattern of ear differences that we have found, while generally compatible with the Morais and Darwin (1974) model, shows a discrepancy in the large ear advantage found for the nonidentical "same" judgments. These judgments also do not show the expected effect of proximity to a phoneme boundary predicted by both models.

\section{REFERENCE NOTES}

1. Repp. B. H. "Posner's paradigm" and categorical perception: A negative study. Status Report on Speech Research. Haskins Laboratories: 1976, SR-45/46, 153-161.

2. Eimas, P. D., \& Miller, J. Auditory memory and the processing of speech. Developmental studies of speech perception: Progress Report No. 3. Providence, R.I: W. S. Hunter Laboratory of Psychology, 1975.

\section{REFERENCES}

Atkinson, R. C., \& Juola, J. F. Factors influencing speed and accuracy of word recognition. In S. Kornblum (Ed.), Attention \& performance IV. New York: Academic Press, 1973.

BAMBER, D. Reaction times and error rates for "same". "different" judgments of multidimensional stimuli. Perception \& Psychophysics, 1969, 6, 169-174.

BAMBER, D. Reaction times and error rates for judging nominal identity of letter strings. Perception \& Psychophysics, 1972, 12, 321-326.

Bamber, D.. \& PAINe, S. Information retrieval processes in "same"-"different" judgments of letter strings. In $S$. Kornblum (Ed.), Attention \& performance IV. New York: Academic Press, 1973.

Besner, D., \& Coltheart, M. Same-different judgments with words and nonwords: The differential effects of relative size. Memory \& Cognition, 1975, 3. 673-677.

Cohen, G., \& Martin, M. Hemispheric differences in an auditory Stroop test. Perception \& Psychophysics, 1975 . 17. 79-83.

Crowder. R. G. The delayed suffix effect following arhythmic stimulus presentation. Quarterly Journal of Experimental Psychology, 1973, 25, 433-439.

Crowder, R. G., \& Morton, J. Precategorical acoustic storage (PAS). Perception \& Psychophysics, 1969, 5. 365-373.

Darwin. C. J., \& Baddeley, A. D. Acoustic memory and the perception of speech. Cognitive Psychology, 1974, 6, 41-60.

Darwin, C. J., Howell. P., \& Brady, S. A. Laterality and localization: A "right ear advantage" for speech heard on the left. In J. Requin (Ed.), Attention \& performance VII. Hillsdale, N.J: Lawrence Erlbaum, in press.

Darwin, C. J., Turvey, M. T., \& Crowder, R. G. An auditory analogue of the Sperling partial report procedure: Evidence for brief auditory storage. Cognitive Psychology. 1972, 3, 255-267.

Fujisaki. H., \& Kawashima, T. The influence of various factors on the identification and discrimination of synthetic speech sounds. Reports of the 6 th International 
Congress on Acoustics. Tokyo, Japan: 1968.

KRUEGER. L. E. Effect of stimulus frequency on speed of same-different judgments. In S. Kornblum (Ed.), Attention \& performance IV. New York: Academic Press, 1973.

Howet.c, P., \& Stockdale, J. E. Memory and display scarch in binary classification reaction time. Perception \& Psychophysics, 1975, 18. 379-388.

Liberman. A. M. Mattingly, I. G., \& Turvey, M. T. Language codes and memory codes. In A. W. Melton \& E. Martin (E.ds.), Coding processes in human memory. New York: Wiley. 1972.

MASSARO. D. M. Preperceptual auditory images. Journal of Experimental Psychology. 1970, 85, 411-417.

Morais. J. Monaural ear differences for same-different reaction times to speech with prior knowledge of ear stimulated. Perceptual and Motor Skills, 1975. 41. 829.830.

Morais. J., \& Darwin, C. J. Ear differences for samedifferent reactions to monaurally presented speech. Brain and Langauge, 1974, 1. 383-390.
Pisoni. D. B. Auditory and phonetic memory codes in the discrimination of consonants and vowels. Perception \& Psychophysics. 1973. 13. 253-260.

Pisoni, D. B.. \& TAsh, J. Reaction times to comparisons within and across phonetic categories. Perception \& Psychophysics, 1974, 15, 285-290.

REPP, B. H. Categorical perception, auditory memory and dichotic interference. Joumal of the Acoustical Society of America, 1975. 57. S51. (Abstract)

Studdert-Kennedy, M. G.. Liberman, A. M., \& Stevens, K. N. Reaction time to synthetic stop consonants and vowels at phoneme centers and at phoneme boundaries. Journal of the Acoustical Society of America, 1963. 35. 1900.

Treisman, M.. \& Rostron, A. B. Brief auditory storage: A modification of Sperling's paradigm applied to audition. Acta Psychologica. 1972. 36. 161-170.

(Received for publication June 1. 1977: accepted July 22. 1977.) 